\title{
Men are from Mars, women are from Venus: Exploring gender differences in personality in the South African context
}

Authors:

Sumaya Laher ${ }^{1}$

Sarah Croxford ${ }^{1}$

Affiliations:

${ }^{1}$ Psychology: School of

Human and Community Development, University of the Witwatersrand South Africa

Correspondence to:

Sumaya Laher

Email:

sumaya.laher@wits.ac.za

Postal address:

Psychology, School of

Human and Community

Development, University

of the Witwatersrand,

Private Bag 3, WITS 2050,

South Africa

Dates:

Received: 04 Oct. 2012

Accepted: 26 July 2013

Published: 02 Oct. 2013

How to cite this article: Laher, S., \& Croxford, S.

(2013). Men are from

Mars, women are from

Venus: Exploring gender

differences in personality in

the South African context. SA

Journal of Human Resource

Management/SA Tydskrif vir

Menslikehulpbronbestuur,

11(1), Art. \#499. 8 pages.

http://dx.doi.org/10.4102/

sajhrm.v11i1.499

\section{Copyright:}

(C) 2013. The Authors.

Licensee: AOSIS

OpenJournals. This work

is licensed under the

Creative Commons

Attribution License.

Read online:
Orientation: Gender differences in personality have been explored in American and European contexts, but African and specifically South African research in the area is lacking.

Research purpose: This study investigated whether there were gender differences in personality and what this might mean for a South African organisational context where personality assessments are frequently employed for decision-making.

Motivation: Personality tests are widely used in many fields, including the industrial, organisational and research fields. Due to the impact that these tests have, it is essential that these tests are used in a fair and unbiased manner.

Research design, approach and method: A cross-sectional, non-experimental design was used. A questionnaire consisting of demographic information and the NEO-PI-R was administered to a non-probability, convenience sample of 425 South African university students. The data was examined using ANOVAs and ANCOVAs.

Main findings: Significant gender differences were found on Neuroticism, Anxiety, Vulnerability, Depression, Self-consciousness, Extraversion, Warmth, Activity, Assertiveness, Positive emotions, Aesthetics, Feelings, Ideas, Agreeableness, Compliance, Tender-mindedness, Altruism, Modesty, Straightforwardness, Trust, Conscientiousness, Order, Achievement striving and Self-discipline.

Practical/managerial implications: The findings indicate differences between men and women are systematic and largely innate and therefore need to be acknowledged when personality tests are used in decision-making. Personality tests also need to be employed constructively to further team-building and diversity.

Contribution/value-add: This study adds to the body of research in South Africa on gender as well as on how the NEO personality scales manifest in different race groups.

\section{Introduction}

Personality tests are widely used in many fields, including research, industrial, organisational, educational and forensic psychology, in general clinical practices and in understanding health risk behaviour (Kaplan \& Saccuzzo, 2009; Moerdyk, 2009). Due to the impact that these tests have on the lives of individuals and the institutions using the tests, it is essential that these psychometric tests provide accurate information about underlying traits. Furthermore, a personality test may contain bias when used in a culture other than the culture in which it was developed (Laher, 2013; Meiring, Van de Vijver, Rothmann \& Barrick, 2005; Van de Vijver \& Leung, 2001; Van Eeden \& Mantsha, 2007). According to the Employment Equity Act (Act No. 55 of 1998), all psychological instruments should be reliable, valid, unbiased and fair for all groups in South Africa where groups are defined by gender and race. Thus gender differences in personality need to be examined. Gender differences are of particular interest because of the implications they may have for bias in decision-making procedures. There is also a documented body of literature internationally that suggests that some gender differences, particularly as they pertain to Neuroticism and Agreeableness, are innate. This presents a separate set of challenges to the organisational context in terms of how these differences should be addressed if they are found to be present in the South African context. Thus, this study explored gender differences across personality traits in a sample of South African university students using the Revised NEO Personality Inventory (NEO-PI-R, Costa \& McCrae, 1992). More specifically, this study examined whether gender differences could be found across the personality domains and facets of the NEO-PI-R. The study also sought to examine if these differences were influenced by race. 


\section{Gender and personality}

As part of a larger study, Feingold (1994) examined gender differences across personality traits in widely used personality tests. Men were found to score moderately higher on Assertiveness $(d=0.50) .{ }^{1}$ Women scored slightly higher on Anxiety $(d=0.25)$ and Trust $(d=0.28)$. The largest difference was found in Tender-mindedness $(d=0.97)$, where women scored higher than men. These patterns were found to be consistent across age, educational level and culture (Feingold, 1994).

Williams, Satterwhite and Best (1999) used the NEO-PI-R to analyse gender stereotypes as perceived by university students across 25 countries. Extraversion, Conscientiousness and Openness to experience were found to be more frequently perceived as male traits, and Neuroticism and Agreeableness more frequently associated with women.

Costa, Terracciano and McCrae (2001) analysed self-report data obtained from the NEO-PI-R across 26 cultures and found consistent patterns of gender differences. They reported the results separately for United States (USA) adults and the other 25 cultures. Among USA adults, women scored higher on Neuroticism $(d=0.51)$, Agreeableness $(d=0.59)$ and Extraversion $(d=0.29)$. These results were replicated in other cultures. The USA sample did not show any significant gender differences for Openness to experience and Conscientiousness. In other countries, however, women scored higher than men on Openness to experience and Conscientiousness, although the effect sizes were very small $(d=0.11-0.16)$ (Costa et al., 2001).

McCrae et al. (2005) found similar results to Costa et al. (2001) when using observer, rather than self-report, ratings obtained from the NEO-PI-R across 50 cultures. Women were found to score higher than men on all scales, with a large effect size for Neuroticism $(d=0.49)$, a small to moderate effect size for Agreeableness $(d=0.32)$, and very small effect sizes for Extraversion $(d=0.15)$, openness $(d=0.07)$ and Conscientiousness $(d=0.14)$.

In contrast to the studies cited above, Hyde (2005) advanced the Gender Similarities Hypothesis which argues that men and women are more similar than different on most psychological variables. Hyde (2005) posits that the magnitude of gender differences are often over-inflated, resulting in the misconception that men and women are vastly different from each other. He argues further that claims about the differences between men and women pervade the mass media, resulting in the development of unsubstantiated gendered stereotypes. Such stereotypes have detrimental effects on relationships, parenting and the advancement of women in the workplace. Hyde (2005) suggested that attention should be paid to effect sizes when interpreting research results with regard to gender differences and proposed that gender differences with an effect size (when calculated using Cohen's $d$ ) of less than 0.35 are too minor to warrant attention.

1.Effect sizes for significant findings are reported using Cohen's $d$.
Feingold (1994) found effect sizes larger than 0.35 on the scales of Tender-mindedness, where women scored higher than men, and Assertiveness, where men scored higher than women. Costa et al. (2001) and McCrae et al. (2005) found that women scored higher than men with an effect size greater than 0.35 on Neuroticism, and Costa et al. (2001) found that women scored higher than men with an effect size greater than 0.35 on the scale of Agreeableness across cultures. Gender differences tend to vary at facet level within the other three domains. For example, in the domain of Extraversion, women typically score higher than men on Warmth and Positive emotions, whereas men typically score higher than women on Assertiveness and Excitement-seeking (Costa et al., 2001; McCrae et al., 2005).

\section{Gender, race and personality}

Costa et al. (2001) and McCrae et al. (2005) found that gender differences tend to be greater in Western cultures than in African cultures (Costa et al., 2001; McCrae et al., 2005). Costa et al. (2001) reported that that Zimbabweans and Black South African people reported very little evidence of gender differences on any of the scales (effect sizes were close to zero on all scales). The biggest gender differences were found in Belgium, with women scoring higher than men on all domains (Neuroticism $d=0.69$, Agreeableness $d=0.55$, Extraversion $d=0.36$ and Openness to experience $d=0.40$ ). White South African people were found to be mid-way in the rank ordering with White women scoring higher than White men on all domains (Neuroticism $d=0.50$, Agreeableness $d=0.46$, Extraversion $d=0.19$ and Openness to experience $d=0.27)$ in Costa et al.'s (2001) study.

McCrae et al. (2005) did not find any significant gender differences in Nigeria, India, Botswana or Ethiopia, and the most significant gender differences were found in the United Kingdom, where women scored higher than men in all domains (Neuroticism $d=0.78$, Agreeableness $d=0.84$, Extraversion $d=0.43$, Openness to experience $d=0.28$ ). South Africa was not included in the McCrae et al. (2005) study.

Given the consistent findings with regard to gender differences, as well as the Employment Equity Act stipulation that tests should be reliable, valid and fair for all groups (where groups are defined by gender and race), this study explored the relationship between race and gender on personality in a South African sample.

\section{Research design Research approach}

This research took the form of a non-experimental, correlational design. There was no manipulation of the independent variables, no random assignment of individuals to groups, and no control group. A questionnaire was administered at a single point in time; hence the design is a cross-sectional design (Whitley, 2002). 


\section{Research method}

\section{Research participants}

The sample consisted of 425 students from the University of the Witwatersrand, Johannesburg. Participants ranged from 18 to 50 years $(M=21.33, S D=3.8)$. Seventy-one percent $(n=303)$ of the sample were female. Forty-eight percent $(n=192)$ were White people and 54.82\% $(n=233)$ were Black people. African, Mixed race and Indian individuals were grouped into the Black people category based on the Employment Equity Act, which collapses these three groups into the Black people grouping. Additionally, research has suggested that Asian and African cultures are typically collectivist and White people (Western cultures) are typically individualist (Eaton \& Louw, 2000; McCrae, 2004). Thus, from a theoretical perspective, combining these groups is also useful. In terms of home language, $68.45 \%(n=291)$ spoke English as their home language and $35.13 \%(n=134)$ spoke another language as their home language.

\section{Measuring instrument}

Data was collected using a self-report questionnaire that incorporated a demographics section and the NEO-PI-R. The demographics section asked participants for their age, gender, race and home language. The NEO-PI-R was developed by Costa and McCrae (1992) as an operationalisation of the fivefactor model (FFM) of personality. Each of the five factors, namely (1) Neuroticism, (2) Extraversion, (3) Openness to experience, (4) Conscientiousness and (5) Agreeableness, is measured individually using 48-item domain scales. Each domain is also made up of six facets, with each facet consisting of eight items. Responses are measured on a five-point Likert scale ranging from strongly disagree $=0$ to strongly agree $=5$.

Research using the NEO-PI-R in South African samples has demonstrated good internal consistency reliability for the domain scales ranging from 0.91 to 0.74 (Laher \& Quy, 2010; Rothman \& Coetzer, 2003; Storm \& Rothman, 2003). In this study, the internal consistency reliability coefficients for the domain scales were 0.91 for Neuroticism, 0.89 for Extraversion, 0.87 for Openness to experience, 0.87 for Agreeableness, and 0.92 for Conscientiousness. With the exception of Actions $(\alpha=0.55)$, Tender-mindedness $(\alpha=0.50)$ and Impulsivity $(\alpha=0.59)$, all the facet scales had internal consistency reliability coefficients above 0.60 .

\section{Procedure}

Ethical clearance for this study was obtained from the Human Research Ethics Committee at the University of the Witwatersrand (Protocol number: HONS/09/0011H). Students were asked to complete the questionnaire during lecture time. Students were informed what completion of the questionnaire entailed. They were assured of confidentiality and anonymity and feedback was available in the form of a summary of the study and results on request. Students placed the completed questionnaires in a sealed box in the lecture room or could return them to a sealed box in the Central Psychology Office. Responses were entered and scored on computer and the relevant statistical analysis was performed using SAS Enterprise Guide (version 4).

\section{Data analysis}

Descriptive statistics using means, standard deviations, minimum and maximum values and skewness coefficients were used for personality scales. Frequencies were conducted for gender and race as reported in the sample section. A one-way analysis of variance (ANOVA), a one-way analysis of co-variance (ANCOVA) and a two-way ANOVA were used to analyse the results. Cohen's $d$ was used to calculate effect sizes for significant pair wise differences whilst eta ${ }^{2}$ was used in the two-way ANOVAs (Huck, 2009).

\section{Results}

Descriptive statistics for the NEO-PI-R are presented first. This is followed by results from the ANOVA analysis exploring gender differences across the NEO-PI-R domain and facet scales. Finally results from the two-way ANOVA and oneway ANCOVA are presented. In the one-way ANCOVA, race is partialled out and in the two-way ANOVA, race and gender are examined together.

\section{Descriptive statistics}

Table 1 presents the means, standard deviations, minimum and maximum values, and skewness coefficients for the

TABLE 1: Descriptive statistics for the NEO-PI-R

\begin{tabular}{|c|c|c|c|c|c|}
\hline Scale & Mean & $\begin{array}{l}\text { Standard } \\
\text { deviation }\end{array}$ & Minimum & Maximum & Skewness \\
\hline Neuroticism & 97.87 & 22.37 & 31 & 160 & 0.12 \\
\hline Anxiety & 18.03 & 5.25 & 0 & 32 & -0.09 \\
\hline Angry hostility & 15.01 & 5.15 & 2 & 29 & 0.22 \\
\hline Depression & 16.92 & 5.87 & 2 & 32 & 0.08 \\
\hline Self-consciousness & 16.93 & 5.23 & 2 & 30 & 0.00 \\
\hline Impulsiveness & 18.22 & 4.35 & 3 & 29 & -0.07 \\
\hline Vulnerability & 12.79 & 4.87 & 0 & 29 & 0.36 \\
\hline Extraversion & 117.23 & 19.84 & 57 & 168 & -0.16 \\
\hline Warmth & 22.93 & 4.42 & 8 & 32 & -0.50 \\
\hline Gregariousness & 17.62 & 5.46 & 2 & 32 & -0.13 \\
\hline Assertiveness & 16.78 & 5.13 & 3 & 30 & -0.10 \\
\hline Activity & 17.91 & 4.22 & 8 & 30 & 0.07 \\
\hline Excitement-seeking & 19.61 & 5.17 & 4 & 32 & -0.25 \\
\hline Positive emotions & 22.36 & 4.96 & 5 & 32 & -0.57 \\
\hline Openness to experience & 122.71 & 18.13 & 70 & 175 & 0.02 \\
\hline Fantasy & 21.07 & 5.12 & 5 & 32 & -0.07 \\
\hline Aesthetics & 20.92 & 5.53 & 4 & 32 & -0.40 \\
\hline Feelings & 22.88 & 4.26 & 10 & 32 & -0.19 \\
\hline Actions & 15.71 & 3.89 & 6 & 28 & 0.04 \\
\hline Ideas & 21.65 & 5.24 & 2 & 32 & -0.32 \\
\hline Values & 20.53 & 4.32 & 4 & 31 & 0.13 \\
\hline Agreeableness & 115.74 & 17.81 & 57 & 161 & -0.37 \\
\hline Trust & 17.03 & 5.19 & 2 & 31 & -0.22 \\
\hline Straightforwardness & 19.17 & 4.54 & 7 & 31 & -0.17 \\
\hline Altruism & 23.18 & 3.83 & 11 & 32 & -0.26 \\
\hline Compliance & 16.50 & 4.67 & 2 & 29 & -0.05 \\
\hline Modesty & 18.61 & 5.19 & 0 & 32 & -0.37 \\
\hline Tender-mindedness & 21.33 & 3.60 & 9 & 29 & -0.37 \\
\hline Conscientiousness & 115.40 & 21.45 & 44 & 174 & -0.36 \\
\hline Competence & 20.52 & 3.92 & 8 & 31 & -0.21 \\
\hline Order & 18.29 & 5.26 & 2 & 31 & -0.29 \\
\hline Dutifulness & 21.74 & 4.21 & 8 & 32 & -0.26 \\
\hline Achievement striving & 19.37 & 4.95 & 4 & 31 & -0.29 \\
\hline Self-discipline & 18.03 & 5.04 & 4 & 31 & -0.19 \\
\hline Deliberation & 17.46 & 4.82 & 0 & 30 & -0.22 \\
\hline
\end{tabular}


domain and facet scales of the NEO-PI-R. It is evident that all the variables were normally distributed. All scales are also generally within the ranges as specified for the NEO-PI-R (Costa \& McCrae, 1992).

\section{One-way ANOVA/ANCOVA: Gender and personality}

Table 2 shows that gender differences, significant at $p<0.01$, were found on the scales of Neuroticism $(d=0.44)$, Anxiety $(d=0.50)$, Depression $(d=0.30)$, Self-consciousness $(d=0.34)$, Vulnerability $(d=0.47)$, Warmth $(d=0.36)$, Positive emotions $(d=0.29)$, Aesthetics $(d=0.39)$, Feelings $(d=0.34)$, Agreeableness $(d=0.25)$, Straightforwardness $(d=0.21)$, Altruism $(d=0.44)$ and Modesty $(d=0.57)$. Gender differences significant at $p<0.05$ were found on the scales of Assertiveness $(d=0.23)$, Ideas $(d=0.24)$, Compliance $(d=0.23)$, Tender-mindedness $(d=0.10)$, Order $(d=0.23)$ and Achievement striving $(d=0.12)$. From the mean scores it is evident that men scored higher than women on Assertiveness and Ideas only. Similar results were evident in the one-way ANCOVA as evidenced in Table 2.

\section{Two-way ANOVA: Gender, race and personality}

Table 3 presents the results from the two-way ANOVA for gender and race on the NEO-PI-R. A significant gender/race groupings interaction effect was found for Activity $\left(\eta^{2}=0.02\right)$, Positive emotions $\left(\eta^{2}=0.01\right)$, Conscientiousness $\left(\eta^{2}=0.01\right)$, Order $\left(\eta^{2}=0.02\right)$ and Self-discipline $\left(\eta^{2}=0.01\right)$, but the effect sizes were quite small. The post hoc tests revealed that White women scored higher than White men on (1) Activity, (2) Positive emotions, (3) Conscientiousness, (4) Order and (5) discipline. White women scored higher than Black women on Activity. Black women scored higher than White men on Order and Self-discipline. Black men scored higher than White men on Order and Self-discipline, as evidenced in Table 4.

It is further evident from Table 3 that main gender effects, significant at $p<0.01$, were found on the scales of Neuroticism $(d=0.43)$, Anxiety $(d=0.50)$, Depression $(d=0.29)$, Selfconsciousness $(d=0.29)$, Vulnerability $(d=0.46)$, Warmth $(d=0.36)$, Positive emotions $(d=0.31)$, Aesthetics $(d=0.39)$, Feelings $(d=0.34)$, Agreeableness $(d=0.55)$, Straightforwardness $(d=0.43)$, Altruism $(d=0.44)$ and Modesty $(d=0.57)$. Main

TABLE 2: ANOVA results for gender on the NEO-PI-R (row 1) and ANCOVA results after partialling out the effects of race on the NEO-PI-R (row 2).

\begin{tabular}{|c|c|c|c|c|c|c|c|c|}
\hline \multirow[t]{2}{*}{ Scale } & \multirow[t]{2}{*}{$d f$} & \multirow[t]{2}{*}{$F$-test } & \multirow[t]{2}{*}{$\chi^{2}$} & \multirow{2}{*}{$\begin{array}{l}p \text {-value } \\
\text { (row 1) }\end{array}$} & \multirow{2}{*}{$\begin{array}{l}p \text {-value } \\
\text { (row 2) }\end{array}$} & \multicolumn{2}{|c|}{ Mean scores } & \multirow[t]{2}{*}{ Cohen's $d$} \\
\hline & & & & & & Male & Female & \\
\hline Neuroticism & 1422 & 16.48 & 16.21 & $<0.0001 * *$ & $0.000 * *$ & 91.06 & 100.62 & 0.44 \\
\hline Anxiety & 1423 & 22.14 & 21.85 & $<0.0001^{* *}$ & $0.000 * *$ & 16.18 & 18.76 & 0.50 \\
\hline Angry hostility & 1421 & 0.31 & 0.275 & 0.5803 & 0.600 & - & - & - \\
\hline Depression & 1422 & 7.75 & 7.631 & $0.0056 * *$ & $0.006^{* *}$ & 15.67 & 17.41 & 0.30 \\
\hline Self-consciousness & 1421 & 10.04 & 10.11 & $0.0016^{* *}$ & $0.002^{* *}$ & 15.67 & 17.43 & 0.34 \\
\hline Impulsiveness & 1421 & 3.18 & 3.02 & 0.0754 & 0.083 & - & - & - \\
\hline Vulnerability & 1423 & 19.50 & 19.24 & $<0.0001^{* *}$ & $0.000^{* *}$ & 11.18 & 13.43 & 0.47 \\
\hline Extraversion & 1 & 0.79 & 0.72 & 0.3772 & 0.396 & - & - & - \\
\hline Warmth & 1423 & 11.16 & 10.93 & $0.0009 * *$ & $0.001^{* *}$ & 21.81 & 23.38 & 0.36 \\
\hline Gregariousness & 1 & 0.59 & 0.55 & 0.4440 & 0.461 & - & - & - \\
\hline Assertiveness & 1421 & 4.54 & 4.64 & $0.0337^{*}$ & $0.032^{*}$ & 17.61 & 16.44 & 0.23 \\
\hline Activity & 1 & 0.34 & 0.26 & 0.5619 & 0.609 & - & - & - \\
\hline Excitement-seeking & 1422 & 1.33 & 1.27 & 0.2496 & 0.261 & - & - & - \\
\hline Positive emotions & 1422 & 7.31 & 7.19 & $0.0072 * *$ & $0.008^{* *}$ & 21.35 & 22.77 & 0.29 \\
\hline Openness to experience & 1422 & 2.72 & 2.50 & 0.10 & 0.115 & - & - & - \\
\hline Fantasy & 1421 & 0.10 & 0.18 & 0.7549 & 0.675 & - & - & - \\
\hline Aesthetics & 1419 & 12.94 & 12.67 & $0.0004 * *$ & $0.000 * *$ & 19.42 & 21.53 & 39.00 \\
\hline Feelings & 1420 & 9.98 & 9.87 & $0.0017 * *$ & $0.002^{* *}$ & 21.86 & 23.29 & 0.34 \\
\hline Actions & 1422 & 1.71 & 1.73 & 0.1916 & 0.189 & - & - & - \\
\hline Ideas & 1 & 4.78 & 5.03 & $0.0294^{*}$ & $0.025^{*}$ & 22.53 & 21.30 & 0.24 \\
\hline Values & 1420 & 1.79 & 1.62 & 0.1815 & 0.204 & - & - & - \\
\hline Agreeableness & 1 & 25.04 & 24.98 & $<0.0001 * *$ & 0.000 & 109.116 & 118.41 & 0.25 \\
\hline Trust & 1422 & 2.89 & 2.79 & 0.0898 & 0.096 & - & - & - \\
\hline Straightforwardness & 1 & 15.87 & 15.73 & $<0.0001^{* *}$ & $0.000^{* *}$ & 17.82 & 19.72 & 0.21 \\
\hline Altruism & 1423 & 16.97 & 16.79 & $<0.0001 * *$ & $0.000 * *$ & 22.0 & 23.66 & 0.44 \\
\hline Compliance & 1420 & 4.41 & 4.71 & 0.0363 & $0.031^{*}$ & 15.75 & 16.80 & 0.23 \\
\hline Modesty & 1422 & 27.83 & 27.89 & $<0.0001^{* *}$ & $0.000 * *$ & 16.58 & 19.53 & 0.57 \\
\hline Tender-mindedness & 1 & 5.63 & 5.63 & $0.0182^{*}$ & $0.018^{*}$ & 20.67 & 21.59 & 0.10 \\
\hline Conscientiousness & 1423 & 3.24 & 3.26 & 0.0724 & 0.071 & - & - & - \\
\hline Competence & 1422 & 0.09 & 0.08 & 0.7628 & 0.782 & - & - & - \\
\hline Order & 1417 & 4.58 & 4.63 & $0.0329 *$ & $0.032 *$ & 17.43 & 18.64 & 0.23 \\
\hline Dutifulness & 1423 & 1.98 & 1.89 & 0.1600 & 0.171 & - & - & - \\
\hline Achievement striving & 1 & 5.44 & 5.37 & $0.0201 *$ & $0.021^{*}$ & 18.49 & 19.72 & 0.12 \\
\hline Self-discipline & 1 & 2.16 & 2.18 & 0.1428 & 0.14 & - & - & - \\
\hline Deliberation & 1422 & 0.30 & 0.40 & 0.5849 & 0.53 & - & - & - \\
\hline
\end{tabular}

* $p<0.05 ;{ }^{* *} p<0.01$

$d f$, degrees of freedom. 


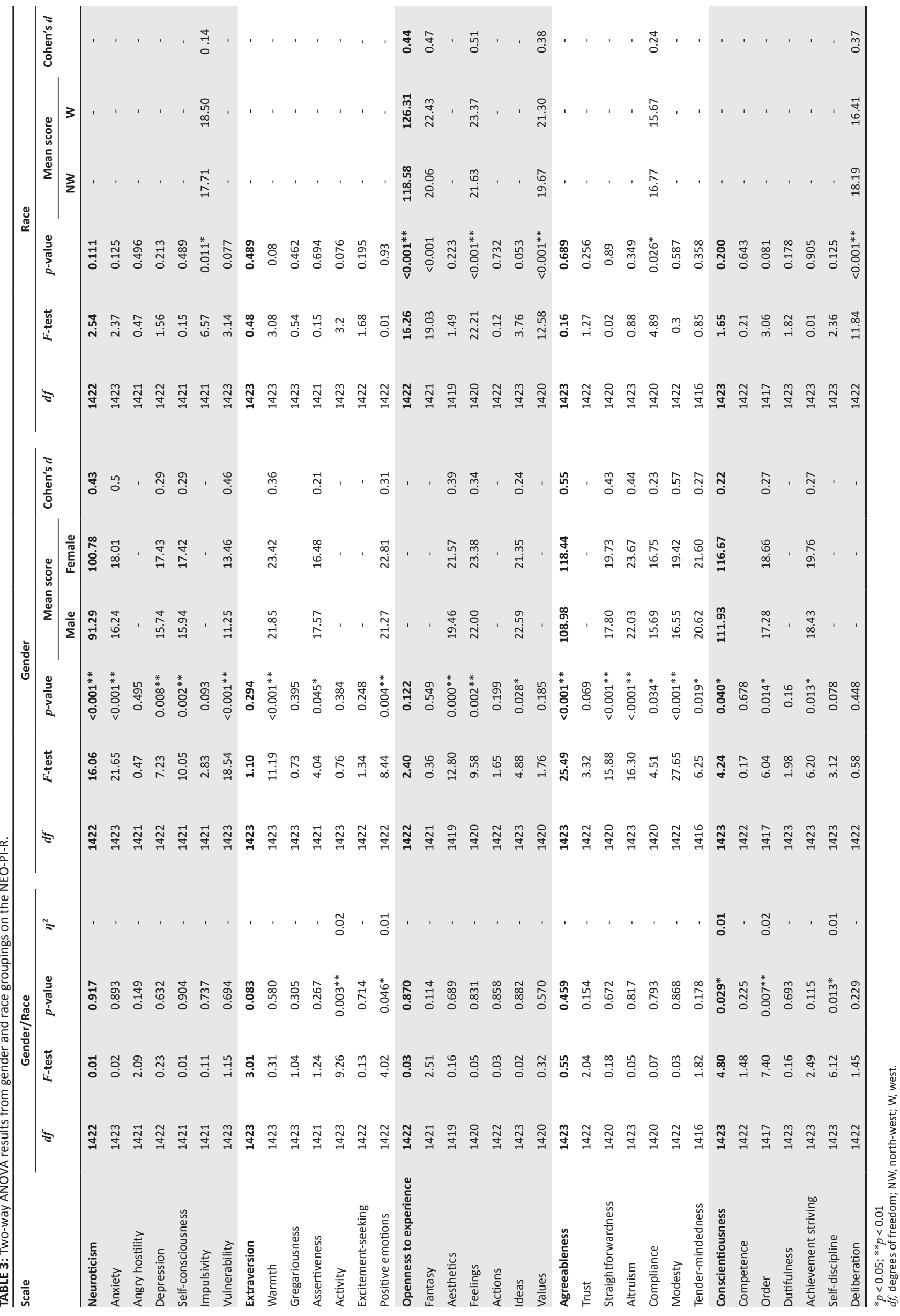


TABLE 4: Post hoc test results from two-way ANOVAs with gender and race groupings on the NEO-PI-R.

\begin{tabular}{|c|c|c|c|c|c|c|}
\hline Gender & Race grouping & Activity & Positive emotions & Conscientiousness & Order & Self-discipline \\
\hline \multirow[t]{2}{*}{ Male } & Black people & 17.96 & 21.82 & 115.93 & 18.53 & 18.40 \\
\hline & White people & 17.41 & 20.71 & 107.93 & 16.02 & 16.23 \\
\hline \multirow[t]{2}{*}{ Female } & Black people & 16.99 & 22.30 & 115.63 & 18.39 & 18.02 \\
\hline & White people & 19.15 & 23.32 & 117.63 & 18.94 & 18.53 \\
\hline
\end{tabular}

gender effects, significant at $p<0.05$, were found on the scales of Assertiveness $(d=0.21)$, Ideas $(d=0.24)$, Compliance $(d=0.23)$, Tender-mindedness $(d=0.27)$, Conscientiousness $(d=0.22)$, Order $(d=0.27)$ and Achievement striving $(d=0.27)$. Examination of the mean scores shows that men scored higher than women on Assertiveness and Ideas only. There were also main race effects that were significant at $p<0.01$. These were for Openness to experience $(d=0.44)$, Fantasy $(d=0.47)$, Feelings $(d=0.51)$, Values $(d=0.38)$ and Deliberation $(d=0.37)$. Significant differences across race at $p<0.05$ were found for impulsiveness $(d=0.14)$ and Compliance $(d=0.24)$.

\section{Discussion}

This study explored gender differences across personality traits using the NEO-PI-R. This was done specifically to investigate implications for bias. Furthermore, race was examined with gender on the personality scales to determine if the pattern of systematic differences would change or not. The findings of this study have the potential to further the understanding of gender bias in personality measurement in the South African context. This is particularly pertinent to the South African context where the Employment Equity Act states that all tests used must be scientifically reliable, valid and fair for all groups, where groups are defined according to gender and race.

Significant gender differences were found on Neuroticism, Anxiety, Vulnerability, Depression, Self-consciousness, Warmth, Activity, Assertiveness, Positive emotions, Aesthetics, Feelings, Ideas, Agreeableness, Compliance, Tendermindedness, Altruism, Modesty, Straightforwardness, Order and Achievement striving. Women scored higher on all scales except for Assertiveness and Ideas, where men scored higher. These results were consistent across the analyses and did not change when the effects of race were included or when they were partialled out.

The pattern of gender differences across the NEO-PI-R facet scales found in this study corresponds most closely to the results from Costa et al.'s (2001) and McCrae et al.'s (2005) adult aged samples, which consistently found that women scored higher than men on the scales of Anxiety, Depression, Self-consciousness, Vulnerability, Warmth, Aesthetics, Feelings, Straightforwardness, Altruism, Modesty and Tender-mindedness, and that men scored higher than women on Assertiveness and Ideas. The results also concur with Feingold's study (1994) in which women scored higher than men on Anxiety and Tender-mindedness, and men scored higher than women on Assertiveness.

Costa et al. (2001) and McCrae et al. (2005) reported that women scored higher than men on Gregariousness, Actions,
Trust and Dutifulness. Costa et al. (2001) also found that women scored higher than men on Angry hostility and impulsiveness and men scored higher than women on Excitement-seeking. These differences were not replicated in this study. In addition, the predicted large effect size for the difference between male and female scores on Assertiveness and Tender-mindedness (Feingold, 1994) were not evident in this study. In contrast to Costa et al.'s (2001) results, which did not indicate a significant gender difference on Achievement striving, the current study found that women scored higher than men on Achievement striving. When using observer rather than self-report measures, McCrae et al. (2005) found that adult men were rated more highly than adult women on Achievement striving, but college-age women were rated higher than college-age men. McCrae et al. (2005) ascribed this to a global increase in the vocational aspirations of young women (McCrae et al., 2005).

There is a body of literature devoted to examining gender differences in personality and various theories are proposed to explain the differences, ranging from evolutionary perspectives to social learning theories; the underlying assumption of all of these theories is that there are fundamental, systematic differences between men and women (see Costa et al., 2001; Friedman \& Schustack, 2009; Hyde, 2005; Larsen \& Buss, 2008). The findings of this study, particularly those differences with moderate effect sizes like Neuroticism, Anxiety, Vulnerability, Aesthetics, Altruism, Modesty and Agreeableness to an extent, lend credence to the argument for certain innate personality traits. This argument is strengthened since this pattern of differences remained invariant across all analyses conducted. Furthermore, it was evident from the results that the interaction between race and gender produced no evidence for systematic differences across White and Black people groupings, suggesting that these differences are invariant across individualist and collectivist cultures.

Costa et al. (2001) alluded to differences in personality expression across gender in individualist versus collectivist cultures, but the results of the current study demonstrate that dividing cultures into those that can be described as individualistic and those that are collectivist is not so clear-cut, especially in the South African context. The possibility exists that using race as a basis for arguments on individualism and collectivism is incorrect. More nuanced ways of examining individualism and collectivism need to be incorporated into further studies.

However, the inclusion of race as variable in the current study did yield some significant findings. Significant differences were observed on Impulsivity, Openness to experience, 
Fantasy, Aesthetics, Values, Compliance and Deliberation across the White people and Black people samples. Black individuals scored lower on all the above-mentioned scales, except for Compliance and Deliberation where they scored higher. Except for the Compliance effect size, all other effect sizes were in the moderate range suggesting that, like gender, race as a variable deserves further investigation on bigger and more representative samples.

\section{Limitations of the study}

Samples drawn from university students are often homogeznous in terms of cultural and social factors and may therefore not be representative of the trends within the larger population (McCrae, 2001). The use of non-probability convenience sampling within a single university population makes it difficult to estimate the degree to which the sample is representative of personality trends in South Africa. The grouping together of African, Mixed race, Indian and Asian individuals to create the Black population grouping was necessary due to the small number of African $(n=121)$, Mixed race $(n=27)$, Indian $(n=76)$ and Asian $(n=9)$ participants who took part in the study. However, collapsing these race groupings into a single group (even though there was a rationale for this) may have obscured differences across personality traits in African, Mixed race, Indian and Asian individuals, and thus negated the ability to accurately to assess the differences among these groups. It is also possible that university samples represent more acculturated samples. It would be necessary therefore for future studies to employ measures of individualism and collectivism as well as acculturation to further understand the nature of gender differences in personality in the South African context.

\section{Implications of this study}

From a more practical perspective, the differences in the mean scores of male and female responses on the NEOPI-R domain and facet scales suggest the possibility of a construct bias when the NEO-PI-R is used in a South African sample. However, one is inclined to question whether the psychometric examination of gender bias in personality instruments is of value, since it is almost a universal finding that men and women will differ on certain characteristics (see Costa et al., 2001; Friedman \& Schustack, 2009; Hyde, 2005).

Either way - whether we examine gender bias in psychometric studies of personality or whether we assume that some differences are universal - we need to address these differences or control for them when we utilise these psychometric instruments. For example, if a man and a woman both apply for the same executive position but it is evident that women generally score lower on Assertiveness, for the woman to get the position she would have to score much higher than women and men alike. To control for this, gender norms become salient and the results of both the current study and previous research indicate that this is warranted.

There is no guarantee that the gender norms created on a standardisation sample in USA can be applied in South
Africa. As is evident from the results of the current study, at the domain level there is sufficient agreement between results found internationally and those in South Africa, but at the facet level there are certain differences.

\section{Implications for human resources management}

To address the issues raised, it would be of value to examine mean differences across gender as standard practice as it will allow one to control for it either by developing local norms, using the norms provided in the test manual if applicable or applying differential selection techniques if applicable. Given that systematic differences have been found across genders in terms of personality, human resources practitioners need to be aware that personality tests cannot be the most reliable factor in selection decisions even if different norms are employed.

On the other hand, personality is an important factor in assessing the person-job fit, and personality (combined with human capital) is argued to be an important predictor of productivity. For example, it is typically assumed that successful managers are ambitious, aggressive and dominant (Weichselbaumer, 2000). The results from the current study suggest that men and women differ on certain personality traits, and that men's high scores on Assertiveness and Ideas might make them more suitable for managerial positions than women.

However, contemporary researchers argue that men and women have both masculine and feminine traits (Maheshwari \& Kumar, 2008). Any individual, regardless of biological sex, may have a feminine orientation, a masculine orientation, or a high degree of masculine and feminine traits, which is referred to as androgyny. Androgynous individuals have been found to be more effective leaders as they are highly flexible in adopting masculine and feminine behaviours as required by the organisational demands. For example, androgynous managers have been found to balance the need for technical capabilities and individual achievement with the need for cohesive group relationships and effective communication.

The above suggests that masculine and feminine attributes are equally valuable in leadership positions. As the sample in this study provided evidence for gender differences across certain personality traits, selecting men and women with their different innate personality traits may facilitate interpersonal learning across genders and the opportunity to develop a more androgynous workforce. Furthermore, the agreeableness and neuroticism differences point to a possibility that women may innately possess a higher EQ, which has proven to be an essential requirement in the current organisational context.

\section{Recommendations for future research}

In order to enhance the understanding of the influence of South African cultural variables on shaping gender differences across personality traits, it may be useful for 
future researchers to investigate the distinctive features of South African culture that shape personality, rather than inferring such influences from changes in personality scores. It may be useful, for example, to investigate the differences in the values held by individuals for whom English is a first language compared to values held by individuals for whom isiZulu is a first language. The use of a sample that is more representative of the South African population would be beneficial in establishing whether the patterns of gender differences in this study can be generalised to the larger South African context. Future research may also consider the use of alternative operationalisations of culture. Hill and Sprague (1999), for example, suggest that social class is an important factor in the socialisation of personality traits. McCrae et al. (2005) suggest that gender differences may be associated with Hofstede's dimensions of individualism, power distance and masculinity. Thus, utilising Hofstede's measure or other measures of individualism and collectivism as well as measures of acculturation would be useful.

\section{Conclusion}

There may be some truth to the adage that men are from Mars and women are from Venus, but as discussed in the preceding sections it is these differences that are so beneficial to an organisation's growth and success. Organisations need to be aware of the potential differences and how best to use them to increase diversity in the workforce and to facilitate team building. The existence of these differences also needs to be acknowledged and addressed in selection and promotion settings to ensure that individuals are not unduly advantaged or disadvantaged based on information from personality tests.

\section{Acknowledgements Competing interest}

The authors declare that they have no financial or personal relationship(s) that may have inappropriately influenced them in writing this article.

\section{Authors' contributions}

S.L. (University of the Witwatersrand) was the project leader and conceptualised the study, collected the data, conducted most of the analysis and wrote majority of the article. S.C. (University of the Witwatersrand) served as a research assistant on the project and assisted with locating some relevant literature for the study, conducting some of the analysis and contributed to writing parts of the literature review and discussion.

\section{References}

Costa, P.T., \& McCrae, R.R. (1992). NEO-PI-R Professional Manual. Florida: Psychological

Assessment Resources, Inc.

Costa, P.T., Terracciano, A., \& McCrae (2001). Gender differences in personality traits across cultures: Robust and surprising findings. Journal of Personality and Social Psychology, 81, 322-331. http://dx.doi.org/10.1037/0022-3514.81.2.322

Eaton, L., \& Louw, J. (2000). Culture and self in South Africa: Individualism - Collectivism predictions. Journal of Social Psychology, 140, 210-217. http://dx.doi.org/10.1080/ 00224540009600461, PMid:10808644

Feingold, A. (1994). Gender differences in personality: A meta-analysis. Psychological Bulletin, 116, 429-456. http://dx.doi.org/10.1037/0033-2909.116.3.429, PMid:7809307

Friedman, H.S., \& Schustack, M.W. (2009. Personality Classic Theories and Modern Research. (4th edn.). Boston: Pearson.

Hill, S.A., \& Sprague, J. (1999). Parenting in Black and White families: The interactions of gender with race and class. Gender and Society, 13, 480-502. http://dx.doi. org/10.1177/089124399013004004

Hyde, J.S. (2005). The gender similarities hypothesis. American Psychologist, 60, 581-592. http://dx.doi.org/10.1037/0003-066X.60.6.581, PMid:16173891

Huck, S. W. (2009). Reading statistics and research (5th edn.). Boston: Pearson.

Kaplan, R.M., \& Saccuzzo, D.P. (2009). Psychological testing: Principles, applications and issues. (7th edn.). Pacific Grove, CA: Brooks/Cole.

Laher, S. (2013). Understanding the Five Factor Model and Five Factor Theory through a South African Cultural Lens. South African Journal of Psychology, 2, 208-221. http://dx.doi.org/10.1177/0081246313483522

Laher, S., \& Quy, G. (2009). Exploring the role of spirituality in the context of the Five Factor Model of personality in a South African sample. Journal of Psychology in Africa, 19(4), 513-521.

Larsen, R.J., \& Buss, D.M. (2008). Personality psychology. (3rd edn.). Boston: McGraw Hill.

Maheshwari, N., \& Kumar, V. (2008). Personal effectiveness as a function of psychological androgyny. Industrial Psychiatry Journal, 17, 39-45.

McCrae, R.R. (2004). Human nature and culture: A trait perspective. Journal of Research in Personality, 38, 3-14. http://dx.doi.org/10.1016/j.jrp.2003.09.009

McCrae, R.R., Terracciano, A. \& 78 Members from the members of the Personality Profiles of Cultures Project. (2005). Universal traits from the observer's perspective: Data from 50 cultures. Journal of Personality and Social Psychology, 88, 547-561. http://dx.doi.org/10.1037/0022-3514.88.3.547

Meiring, D., Van de Vijver, F., Rothmann, S., \& Barrick, M.R. (2005). Construct, item and method bias of cognitive and personality tests in South Africa. South African Journal of Industrial Psychology, 31, 1-8.

Moerdyk, A. (2009). The principles and practice of psychological assessment. Pretoria: Van Schaik Publishers.

Rothman, S., \& Coetzer, E.P. (2003). The Big Five personality dimensions and job performance. South African Journal of Industrial Psychology, 29, 68-74.

Storm, K., \& Rothmann, S. (2003). The relationship between burnout, personality traits and coping strategies in a corporate pharmaceutical group. South African Journal of Industrial Psychology, 29, 35-42.

Van de Vijver, R.J.R., \& Leung, K. ( 2001). Personality in cultural context: Methodological issues. Journal of Personality, 69, 1007-1031. http://dx.doi.org/10.1111/1467issues. Journal of Personality,
6494.696173, PMid:11767816

Van Eeden, R., \& Mantsha, T. (2007). Theoretical and methodological considerations in the translation of the 16PF5 into an African language. South African Journal of Psychology, 37, 62-81. http://dx.doi.org/10.1177/008124630703700105

Weichselbaumer, D. (May, 2000). Is it Sex or Personality? The Impact of Sex-Stereotypes on Discrimination in Applicant Selection. University of Linz Economics Working Paper No. 0021. Retrieved 25 August 2012, from http://www.economics.uni-linz. ac.at/papers/2000/wp0011.pdf

Williams, J.E, Satterwhite, R.C., \& Best, D.L. (1999). Pancultural gender stereotypes revisited: The Five-Factor Model. Sex Roles, 40, 513-525. http://dx.doi.org/10.1023/ A:1018831928829

Whitley, B.E. (2002). Principles of Research and Behavioural Science. Boston: McGraw Hill. 